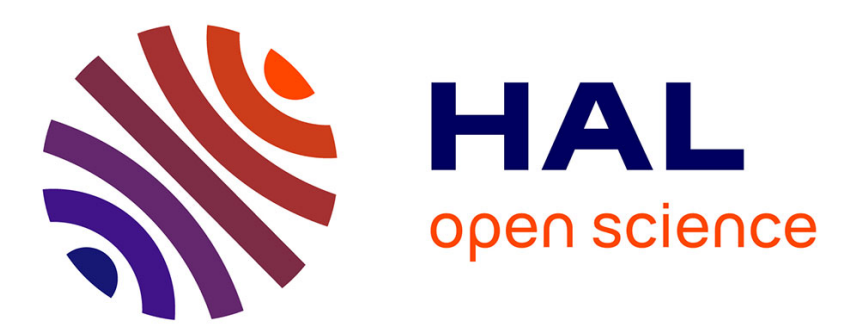

\title{
Efficient pricing of swing options in Lévy-driven models
}

Oleg Kudryavtsev, Antonino Zanette

\section{To cite this version:}

Oleg Kudryavtsev, Antonino Zanette. Efficient pricing of swing options in Lévy-driven models. Quantitative Finance, 2013, 13 (4), pp.627-635. 10.1080/14697688.2012.717708 . hal-00918582

\section{HAL Id: hal-00918582 \\ https://hal.science/hal-00918582}

Submitted on 17 Dec 2013

HAL is a multi-disciplinary open access archive for the deposit and dissemination of scientific research documents, whether they are published or not. The documents may come from teaching and research institutions in France or abroad, or from public or private research centers.
L'archive ouverte pluridisciplinaire HAL, est destinée au dépôt et à la diffusion de documents scientifiques de niveau recherche, publiés ou non, émanant des établissements d'enseignement et de recherche français ou étrangers, des laboratoires publics ou privés. 


\title{
Efficient pricing of swing options in Lévy-driven models
}

\author{
Oleg Kudryavtsev* \\ Department of Informatics, \\ Russian Customs Academy Rostov Branch, \\ Budennovskiy 20, Rostov-on-Don, 344002, Russia \\ koe@donrta.ru \\ Antonino Zanette \\ Dipartimento di Finanza dell'Impresa \\ e dei Mercati Finanziari \\ Università di Udine \\ via Tomadini 30/A, Udine, Italy \\ antonino.zanette@uniud.it
}

\begin{abstract}
We consider the problem of pricing swing options with multiple exercise rights in Lévy-driven models. We propose an efficient Wiener-Hopf factorisation method that solves multiple parabolic partial integro-differential equations associated with the pricing problem. We compare the proposed method with a finite difference algorithm. Both proposed deterministic methods are related to the dynamic programming principle and lead to the solution of a multiple optimal stopping problem. Numerical examples illustrate the efficiency and the precision of the proposed methods.
\end{abstract}

Keywords: Option pricing; swing options; Finite difference methods; Wiener-Hopf factorisation; American options; energy derivatives; Numerical methods for option pricing.

\section{Introduction}

The motivation for this work comes from energy markets, where financial instruments are increasingly important for risk management. In a deregulated market, energy contracts will need to be priced according to their financial risk. Due to the uncertainty of consumption and the limited fungibility of energy, new financial contracts such as swing options have been introduced in the commodity market. Swing options are an American type of options with many exercise rights. Their owner can exercise them at many times under the condition that they respect the refracting time that separates two

\footnotetext{
${ }^{*}$ Corresponding author. Email: koe@donrta.ru
} 
successive exercises. Swing options are also widely used in the gas and oil markets. Thus, pricing swing options will be increasingly important.

Many numerical methods, essentially based on the solution to the dynamic programming equation, have been introduced recently in the financial literature. In the context of swing options, two different probabilistic strategies have been developed. In the first, swing options are priced using an extension of the binomial tree algorithm, leading to the so-called forest tree (Lari-Lavassani et al. (2001), Jaillet et al. (2004)). In the second, Monte Carlo methods are used, in which conditional expectations are computed using either regression techniques (Barrera-Esteve et al. (2006)) or Malliavin calculus (Mnif and Zhegal (2006), Carmona and Touzi (2008)). In particular, Carmona and Touzi (2008) propose a Monte Carlo approach to the problem of pricing American put options, in a finite time horizon, with multiple exercise rights in the case of geometric Brownian motion. In that paper, they introduce the inductive hierarchy of Snell envelopes needed in the multiple exercise case.

Energy expenditure increases sharply with higher daily temperature variation, and consequently, price varies. Although these spikes of power consumption are infrequent, they have a large financial impact, and therefore many authors propose to price swing options in a model with jumps (see Mnif and Zhegal (2006), Wilhelm and Winter (2008)). Mnif and Zhegal (2006) extend the results of Carmona and Touzi (2008) to a market with jumps. In fact, the multiple stopping time problem for swing options can be reduced to a cascade of single stopping time problems in a Lévy market where jumps are permitted. With regards to deterministic methods, Wilhelm and Winter (2008) develop a finite element algorithm for pricing swing options in different models, including jump models.

Boyarchenko and Levendorskii (2006) apply the Wiener-Hopf method to similar but distinct multistage investment or disinvestment problems (sequences of embedded perpetual American (real) options) under uncertainty modelled as a monotone function of a Lévy process; in the case of the Kou model, closed form solutions are given.

In this paper, we propose two approaches to solving multiple parabolic partial integro-differential equations (PIDEs) for pricing swing options in jump models. The first method, which is very simple and is introduced for comparison purposes, uses a finite difference scheme to solve the system of variational inequalities associated with the swing option problem approached through the splitting method proposed in Barles et al. (1995).

The second method uses Fast Wiener-Hopf factorisation (FWHF), introduced in Kudryavtsev and Levendorskii (2009), where a fast and accurate numerical method for pricing barrier option for a wide class of Lévy processes was constructed. The FWHF method is based on an efficient approximation of the Wiener-Hopf factors and the Fast Fourier Transform algorithm. The advantage of the WienerHopf approach over finite difference schemes in terms of accuracy and convergence was shown in Kudryavtsev and Levendorskii (2009). We will propose here a new efficient pricing algorithm for swing options that involves dynamic programming and the solving of multiple PIDEs by the FWHFmethod. We apply this algorithm for pricing swing options where the spot electricity price is a Lévy process that allows the consideration of jump risk. Numerical results, developed as in Wilhelm and Winter (2008) in the Black-Scholes and CGMY models, show the efficiency and the accuracy of the 
proposed algorithms.

The rest of the paper is organised as follows. Section 2 is devoted to the basic facts on Lévy processes. In Section 3, we present the multiple optimal stopping problem for swing options. In Section 4 and Section 5, we propose, respectively, a finite difference and a Wiener-Hopf approach for pricing swing options. The numerical results are presented in Section 6.

\section{Lévy processes: basic facts}

\subsection{General definitions}

A Lévy process is a stochastically continuous process with stationary independent increments (for general definitions, see, e.g., Sato (1999)). A Lévy process may have a Gaussian component and/or a pure jump component. The latter is characterised by the density of jumps, which is called the Lévy density. A Lévy process $X_{t}$ can be completely specified by its characteristic exponent, $\psi$, definable from the equality $E\left[e^{i \xi X(t)}\right]=e^{-t \psi(\xi)}$ (we confine ourselves to the one-dimensional case).

The characteristic exponent is given by the Lévy-Khintchine formula

$$
\psi(\xi)=\frac{\sigma^{2}}{2} \xi^{2}-i \mu \xi+\int_{-\infty}^{+\infty}\left(1-e^{i \xi y}+i \xi y \mathbf{1}_{|y| \leq 1}\right) \nu(d y)
$$

where $\sigma^{2} \geq 0$ is the variance of the Gaussian component, and the Lévy measure $\nu(d y)$ satisfies

$$
\int_{\mathbb{R} \backslash\{0\}} \min \left\{1, y^{2}\right\} \nu(d y)<+\infty .
$$

Assume that under a risk-neutral measure chosen by the market, the price process has the dynamics $S_{t}=e^{X_{t}}$, where $X_{t}$ is a certain Lévy process. Then we must have $E\left[e^{X_{t}}\right]<+\infty$, and, therefore, $\psi$ must admit analytic continuation into a strip $\Im \xi \in(-1,0)$ and continuous continuation into the closed strip $\Im \xi \in[-1,0]$.

The infinitesimal generator of $X$, denoted by $L$, is an integro-differential operator that acts as follows:

$$
L u(x)=\frac{\sigma^{2}}{2} \frac{\partial^{2} u}{\partial x^{2}}(x)+\mu \frac{\partial u}{\partial x}(x)+\int_{-\infty}^{+\infty}\left(u(x+y)-u(x)-y \mathbf{1}_{|y| \leq 1} \frac{\partial u}{\partial x}(x)\right) \nu(d y) .
$$

The infinitesimal generator $L$ also can be represented as a pseudo-differential operator (PDO) with the symbol $-\psi(\xi)$, i.e., $L=-\psi(D)$, where $D=-i \partial_{x}$. Recall that a PDO $A=a(D)$ acts as follows:

$$
A u(x)=(2 \pi)^{-1} \int_{-\infty}^{+\infty} e^{i x \xi} a(\xi) \hat{u}(\xi) d \xi
$$

where $\hat{u}$ is the Fourier transform of a function $u$

$$
\hat{u}(\xi)=\int_{-\infty}^{+\infty} e^{-i x \xi} u(x) d x
$$


Note that the inverse Fourier transform in (2.4) is defined in the classical sense only if the symbol $a(\xi)$ and function $\hat{u}(\xi)$ are sufficiently nice. In general, one defines the (inverse) Fourier transform by duality.

Further, if the riskless rate, $r$, is constant, and if the stock does not pay dividends, then the discounted price process must be a martingale. Equivalently, the following condition (the EMMrequirement) must hold (see, e.g., Boyarchenko and Levendorskii (2002)):

$$
r+\psi(-i)=0
$$

which can be used to express $\mu$ via the other parameters of the Lévy process, as below:

$$
\mu=r-\frac{\sigma^{2}}{2}+\int_{-\infty}^{+\infty}\left(1-e^{y}+y \mathbf{1}_{|y| \leq 1}\right) \nu(d y) .
$$

Hence, the infinitesimal generator may be rewritten as follows:

$$
L u(x)=\frac{\sigma^{2}}{2} \frac{\partial^{2} u}{\partial x^{2}}(x)+\left(r-\frac{\sigma^{2}}{2}\right) \frac{\partial u}{\partial x}(x)+\int_{\mathbb{R}}\left[u(x+y)-u(x)-\left(e^{y}-1\right) \frac{\partial u}{\partial x}(x)\right] \nu(d y) .
$$

\subsection{Regular Lévy processes of exponential type}

Loosely speaking, a Lévy process $X$ is called a Regular Lévy Process of Exponential type (RLPE) if its Lévy density has a polynomial singularity at the origin and decays exponentially at infinity (see Boyarchenko and Levendorskii (2002))). A nearly equivalent definition is as follows: the characteristic exponent is analytic in a strip $\Im \xi \in\left(\lambda_{-}, \lambda_{+}\right), \lambda_{-}<-1<0<\lambda_{+}$, is continuous up to the boundary of the strip, and admits the representation

$$
\psi(\xi)=-i \mu \xi+\phi(\xi)
$$

where $\phi(\xi)$ stabilises to a positively homogeneous function at infinity:

$$
\phi(\xi) \sim c_{ \pm}|\xi|^{\nu}, \quad \text { as } \Re \xi \rightarrow \pm \infty, \quad \text { in the strip } \Im \xi \in\left(\lambda_{-}, \lambda_{+}\right),
$$

where $c_{ \pm}>0$. "Nearly" means that the majority of classes of Lévy processes used in empirical studies of financial markets satisfy the conditions of both definitions. These classes are as follows: Brownian motion, Kou's model (Kou (2002)), Hyperbolic processes (Eberlein and Keller (1995)), Normal Inverse Gaussian processes and their generalisation (Barndorff-Nielsen (1998) and BarndorffNielsen and Levendorskii (2001)), and the extended Koponen's family. Koponen (1995) introduced a symmetric version; Boyarchenko and Levendorskii (2000) gave a non-symmetric generalisation; later, a subclass of this model appeared under the name CGMY - model in Carr et al. (2002), and Boyarchenko and Levendorskii (2002) used the name KoBoL family.

The important exception is Variance Gamma Processes (VGP; see, e.g., Madan et al. (1998)). VGP satisfy the conditions of the first definition but not the second one, as the characteristic exponent behaves like const $\cdot \ln |\xi|$, as $\xi \rightarrow \infty$. 
Example 2.1. The characteristic exponent of a pure jump CGMY model is given by

$$
\psi(\xi)=-i \mu \xi+C \Gamma(-Y)\left[G^{Y}-(G+i \xi)^{Y}+M^{Y}-(M-i \xi)^{Y}\right]
$$

where $C>0, \mu \in \mathbb{R}, Y \in(0,2), Y \neq 1$, and $-M<-1<0<G$.

Example 2.2. If the Lévy measure of a jump diffusion process is given by a normal distribution

$$
\nu(d x)=\frac{\lambda}{\delta \sqrt{2 \pi}} \exp \left(-\frac{(x-\gamma)^{2}}{2 \delta^{2}}\right) d x
$$

then we obtain the Merton model. The parameter $\lambda$ characterises the intensity of jumps. The characteristic exponent of the process is of the form

$$
\psi(\xi)=\frac{\sigma^{2}}{2} \xi^{2}-i \mu \xi+\lambda\left(1-\exp \left(-\frac{\delta^{2} \xi^{2}}{2}+i \gamma \xi\right)\right)
$$

where $\sigma, \delta, \lambda \geq 0, \mu, \gamma \in \mathbb{R}$.

There are two important degenerate cases:

- If the intensity of jumps $\lambda=0$, then we obtain Black-Scholes model with $\mu=r-\frac{\sigma^{2}}{2}$ fixed by the EMM-requirement;

- If the intensity of jumps $\lambda>0$ but $\delta=0$, then we obtain a jump diffusion process with a constant jump size $\gamma$; the drift term $\mu=r-\frac{\sigma^{2}}{2}+\lambda\left(1-e^{\gamma}\right)$ is fixed by the EMM-requirement.

\subsection{The Wiener-Hopf factorisation}

There are several forms of the Wiener-Hopf factorisation. The Wiener-Hopf factorisation formula used in probability reads as follows:

$$
E\left[e^{i \xi X_{T}}\right]=E\left[e^{i \xi \bar{X}_{T}}\right] E\left[e^{i \xi \underline{X}_{T}}\right], \quad \forall \xi \in \mathbb{R}
$$

where $T \sim \operatorname{Exp} q$, and $\bar{X}_{t}=\sup _{0 \leq s \leq t} X_{s}$ and $\underline{X}_{t}=\inf _{0 \leq s \leq t} X_{s}$ are the supremum and infimum processes. Introducing the notation

$$
\begin{aligned}
& \varphi_{q}^{+}(\xi)=q E\left[\int_{0}^{\infty} e^{-q t} e^{i \xi \bar{X}_{t}} d t\right]=E\left[e^{i \xi \bar{X}_{T}}\right], \\
& \varphi_{q}^{-}(\xi)=q E\left[\int_{0}^{\infty} e^{-q t} e^{i \xi \underline{X}_{t}} d t\right]=E\left[e^{i \xi \underline{X}_{T}}\right]
\end{aligned}
$$

we can write $(2.12)$ as

$$
\frac{q}{q+\psi(\xi)}=\varphi_{q}^{+}(\xi) \varphi_{q}^{-}(\xi)
$$

Equation (2.15) is a special case of the Wiener-Hopf factorisation of the symbol of a PDO. In applications to Lévy processes, the symbol is $q /(q+\psi(\xi))$, and the PDO is $\mathcal{E}_{q}:=q /(q-L)=q(q+\psi(D))^{-1}$ : the normalised resolvent of the process $X_{t}$ or, using the terminology of Boyarchenko and Levendorskii 
(2005), the expected present value operator (EPV-operator) of the process $X_{t}$. The name is due to the observation that, for a stream $g\left(X_{t}\right)$,

$$
\mathcal{E}_{q} g(x)=E\left[\int_{0}^{+\infty} q e^{-q t} g\left(X_{t}\right) d t \mid X_{0}=x\right] .
$$

We introduce the following operators:

$$
\mathcal{E}_{q}^{ \pm}:=\varphi_{q}^{ \pm}(D),
$$

which also admit interpretation as the EPV-operators under supremum and infimum processes. One of the basic observations in the theory of PDO is that the product of symbols corresponds to the product of operators. In our case, it follows from (2.15) that

$$
\mathcal{E}_{q}=\mathcal{E}_{q}^{+} \mathcal{E}_{q}^{-}=\mathcal{E}_{q}^{-} \mathcal{E}_{q}^{+}
$$

as operators in appropriate function spaces.

For a wide class of Lévy models, $\mathcal{E}$ and $\mathcal{E}^{ \pm}$admit interpretation as expectation operators:

$$
\mathcal{E}_{q} g(x)=\int_{-\infty}^{+\infty} g(x+y) P_{q}(y) d y, \quad \mathcal{E}_{q}^{ \pm} g(x)=\int_{-\infty}^{+\infty} g(x+y) P_{q}^{ \pm}(y) d y,
$$

where $P_{q}(y), P_{q}^{ \pm}(y)$ are certain probability densities with

$$
P_{q}^{ \pm}(y)=0, \forall \pm y<0 .
$$

Moreover, the characteristic functions of the distributions $P_{q}(y)$ and $P_{q}^{ \pm}(y)$ are $q(q+\psi(\xi))^{-1}$ and $\varphi_{q}^{ \pm}(\xi)$, respectively.

The general results in this paper are based on simple properties of the EPV operators, which follow immediately from the interpretation of $\mathcal{E}^{ \pm}$as expectation operators. For details, see Boyarchenko and Levendorskiï (2005).

Proposition 2.1 EPV-operators $\mathcal{E}_{q}^{ \pm}$have the following properties

1. If $g(x)=0 \forall x \geq h$, then $\forall x \geq h,\left(\mathcal{E}_{q}^{+} g\right)(x)=0$ and $\left(\left(\mathcal{E}_{q}^{+}\right)^{-1} g\right)(x)=0$.

2. If $g(x)=0 \forall x \leq h$, then $\forall x \leq h,\left(\mathcal{E}_{q}^{-} g\right)(x)=0$ and $\left(\left(\mathcal{E}_{q}^{-}\right)^{-1} g\right)(x)=0$.

3. If $g(x) \geq 0 \forall x$, then $\left(\mathcal{E}_{q}^{+} g\right)(x) \geq 0, \forall x$. If, in addition, there exists $x_{0}$ such that $g(x)>0$ $\forall x>x_{0}$, then $\left(\mathcal{E}_{q}^{+} g\right)(x)>0 \forall x$.

4. If $g(x) \geq 0 \forall x$, then $\left(\mathcal{E}_{q}^{-} g\right)(x) \geq 0, \forall x$. If, in addition, there exists $x_{0}$ such that $g(x)>0$ $\forall x<x_{0}$, then $\left(\mathcal{E}_{q}^{-} g\right)(x)>0 \forall x$.

5. If $g$ is monotone, then $\mathcal{E}_{q}^{+} g$ and $\mathcal{E}_{q}^{-} g$ are also monotone.

6. If $g$ is continuous and satisfies

$$
|g(x)| \leq C\left(e^{\sigma_{-} x}+e^{\sigma_{+} x}\right), \quad \forall x \in \mathbb{R},
$$

where $\sigma_{-} \leq 0 \leq \sigma_{+}$and $C$ are all independent of $x$, then $\mathcal{E}_{q}^{+} g$ and $\mathcal{E}_{q}^{-} g$ are continuous. 


\section{The multiple optimal stopping problem for swing options}

We consider a price process that evolves according to the formula

$$
S_{t}=e^{X_{t}}
$$

where $\{X\}_{t \geq 0}$, the driving process, is an adapted Lévy process defined on the filtered probability space $\left(\Omega, \mathcal{F}, \mathbb{F}=\left\{\mathcal{F}_{t}\right\}_{t \geq 0}, \mathbb{P}\right)$, satisfying the usual conditions.

Let $T$ be the option's maturity time, and let $\mathcal{T}_{t, T}$ be the set of $\mathbb{F}$-stopping times with values in $[t, T]$. Consider a swing option that gives the right to multiple exercise with $\delta>0$ refracting period that separates two successive exercises (the number of possible exercises is fixed). We consider the possibility of $n$ put exercises. We shall denote by $\mathcal{T}^{n}$ the collection of all vectors of stopping times $\left(\tau_{1}, \tau_{2}, \ldots, \tau_{n}\right)$, such that

- $\tau_{1} \leq T$ a.s.

- $\tau_{i}-\tau_{i-1} \geq \delta$ on $\left\{\tau_{i-1} \leq T\right\}$ a.s., for all $i=2, . ., n$

Denote by $v^{(i)}(t, x)$ the swing option value with the possibility of $i$ exercises at spot level $S=e^{x}$ and time $t \leq T$. Following [12], the multiple exercise problem can be solved computing

$$
v^{(n)}(0, x)=\sup _{\left(\tau_{1}, \ldots, \tau_{n}\right) \in \mathcal{T}^{n}} \sum_{i=1}^{n} E\left[e^{-r \tau_{i}} \phi\left(X_{\tau_{i}}\right)\right]
$$

where

$$
\phi(x)=\left(K-e^{x}\right)_{+}
$$

is the payoff function.

To solve the multiple optimal stopping problem, Carmona and Touzi (2008) introduce the idea of a inductive hierarchy. In fact, they reduce the multiple stopping problem to a cascade of $n$ optimal single stopping problems. Define the value function for $i=1, \ldots, n$

$$
v^{(i)}(t, x)=\sup _{\tau \in \mathcal{T}_{t, T}} E\left[e^{-r \tau} \phi^{(i)}\left(\tau, X_{\tau}^{t, x}\right)\right]
$$

where the reward function $\phi^{(i)}$ is now defined as

$$
\begin{array}{r}
\phi^{(i)}(t, x)=\phi(x)+E\left[e^{-r \delta} v^{(i-1)}\left(t+\delta, X_{t+\delta}^{t, x}\right)\right], \quad t \leq T-\delta \\
\phi^{(i)}(t, x)=\phi(x), \quad t>T-\delta .
\end{array}
$$

The problem could be solved using a Monte Carlo algorithm. Let $t_{0}=0<t_{1}<t_{2}<\ldots<t_{N}=T$ be a time discretisation grid. The price of a swing option can be computed by the backward induction procedure

$$
\left\{\begin{array}{l}
v^{(i)}\left(t_{N}, x\right)=\phi(x) \\
v^{(i)}\left(t_{k-1}, x\right)=\max \left\{\phi^{(i)}\left(t_{k-1}, x\right) ; e^{-r\left(t_{k}-t_{k-1}\right)} E\left[v^{(i)}\left(t_{k}, X_{t_{k}}^{t_{k-1}, x}\right)\right]\right\}, k=N, \ldots, 1 .
\end{array}\right.
$$


Carmona-Touzi (2008) and Mnif-Zeghal (2006), respectively, considered a Monte Carlo Malliavinbased algorithm to compute the price in the Black-Scholes and jump models frameworks. BarreraEsteve et al. (2006) used a regression based method to approximate conditional expectations. In the next sections, we propose two PIDE-based approaches.

\section{The finite difference scheme for pricing swing options}

We can compute the swing option price using the formulation given in (3.2) with an analytical approach. In fact, we propose to solve the following system of variational inequalities associated with the swing options formulation

$$
\left\{\begin{array}{l}
\max \left(\phi^{(i)}(t, x)-v^{(i)}(t, x), \frac{\partial v^{(i)}}{\partial t}+L v^{(i)}-r v^{(i)}\right)=0,(t, x) \text { in }[0, T[\times \mathbb{R}, \\
v^{(i)}(T, x)=\phi^{(i)}\left(T, e^{x}\right) .
\end{array}\right.
$$

with $i=1, \ldots, n$, where the integro-differential operator $L$ is defined in (2.7).

Now recall that for $t \leq T-\delta$

$$
\phi^{(i)}(t, x)=\left(K-e^{x}\right)_{+}+E\left[e^{-r \delta} v^{(i-1)}\left(t+\delta, X_{t+\delta}^{t, x}\right)\right] .
$$

Let us define for $t \leq T-\delta$

$$
u^{(i)}(t, x)=E\left[e^{-r \delta} v^{(i)}\left(t+\delta, X_{t+\delta}^{t, x}\right)\right] .
$$

By the Feyman-Kac theorem, $u^{(i)}(t, x)=z(0, x)$, where $z(t, x)$ is the solution of the following partial integro-differential equation (PIDE):

$$
\left\{\begin{array}{l}
\frac{\partial z}{\partial t}+L z-r z=0,(t, x) \in[0, \delta[\times \mathbb{R} \\
z(\delta, x)=v^{(i)}(t+\delta, x)
\end{array}\right.
$$

which can be numerically computed using a finite difference approach. To price a swing option, therefore, we can solve the system of variational inequalities (4.1) computing the reward payoff function $\phi^{(i)}(t, x)$ in the following way:

$$
\phi^{(i)}(t, x)=\phi(x)
$$

for $T-\delta<t \leq T$, and

$$
\phi^{(i)}(t, x)=\left(K-e^{x}\right)_{+}+u^{(i-1)}(t, x)
$$

for $t \leq T-\delta$.

As stated above, the reward payoff function can be computed numerically using a finite difference scheme. The numerical solution of the variational inequalities (4.1) requires numerically solving each PIDE problem (4.2). To solve (4.1) and (4.2), we perform the following steps:

- Localisation. We choose a spatial bounded computational domain $\Omega_{l}$, which implies that we must choose some artificial boundary conditions. 
- Truncation of large jumps. This step corresponds to truncating the integration domain in the integral part.

- Discretisation. The derivatives of the solution are replaced by finite differences, and the integral terms are approximated using the trapezoidal rule. Then the problem is solved by using an explicit-implicit scheme (see Briani et al (2004), Cont and Voltchkova (2005) and its program implementation, PREMIA). In particular, we introduce a time grid $t=s \Delta t, s=0, . ., N$, where $\Delta t=\frac{T}{N}$ is the time step. At each time step, it is necessary to solve a linear system for the linear problem (4.2) and a linear complementarity problem for the nonlinear problem (4.1).

The idea of the explicit-implicit method is based on an asymmetric treatment of the differential and integral parts of $L$. The operator $L$ in (4.2) is split into two parts

$$
L z=D z+J z
$$

where $D$ and $J$ are the differential and integral parts of $L$, respectively. We replace $D z$ with a finite difference approximation $D_{\Delta} z$ and $J z$ with the trapezoidal quadrature approximation $J_{\Delta} z$ and use the following explicit-implicit time-stepping:

$$
\frac{z^{s+1}-z^{s}}{\Delta t}+D_{\Delta} z^{s}++J_{\Delta} z^{s+1}-r z^{s}=0
$$

The integral part is treated in explicit way to avoid a dense matrix, while the differential part is treated in implicit way. Details of the algorithms are given in Cont and Voltchkova (2005).

- Treatment of the variational inequalities. We solve each of the variational inequalities (4.1) using the splitting method of Barles et al (1995). The splitting methods can be viewed as an analytical version of dynamic programming. The idea of this scheme is to split the American problem into two steps: we construct recursively the approximate solution $v^{(i)}(s \Delta t, x)$ at each time step $s \Delta t$ by starting from $v^{(i)}(N \Delta t, x)=\phi(x)$ and computing at each time step the values of $v^{(i)}(s \Delta t, x)$ for $s=N-1, . ., 0$ as follows:

- Compute the solution of the following linear Cauchy problem on $\left[s \Delta t,(s+1) \Delta t\left[\times \Omega_{l}\right.\right.$ using an explicit-implicit scheme:

$$
\left\{\begin{array}{l}
\frac{\partial w^{(i)}(s \Delta t, x)}{\partial t}+L w^{(i)}(s \Delta t, x)-r w^{(i)}(s \Delta t, x)=0, \quad \text { in }\left[s \Delta t,(s+1) \Delta t\left[\times \Omega_{l}\right.\right. \\
w^{(i)}((s+1) \Delta t, x)=v^{(i)}((s+1) \Delta t, x)
\end{array}\right.
$$

- Apply the early exercise $v^{(i)}(s \Delta t, x)=\max \left(w^{(i)}(s \Delta t, x), \phi^{(i)}(s \Delta t, x)\right)$, where the reward function $\phi^{(i)}(s \Delta t, x)$ is obtained by solving the linear problem (4.2) with an explicit-implicit finite difference method.

One could also apply the method of horizontal lines or the Carr's randomisation to (3.2), then use the explicit-implicit finite difference scheme to solve the corresponding sequence of free boundary problems. The analytical method of lines was introduced to finance by Carr and Faguet (1994); Carr 
(1998) suggested an important new probability interpretation of the method, which we call Carr's randomisation. In the case of American options, the convergence of Carr's randomisation algorithm is proved in Bouchard et al. (2005) for a wide class of strong Markov processes. In the next section, we will start with Carr's randomisation procedure.

\section{Pricing swing options using the Wiener-Hopf approach}

In this section, we apply the Wiener-Hopf approach to pricing swing options. The first step is to discretise the time $(0=) t_{0}<t_{1}<\cdots<t_{N}(=T)$ but not the space variable. Set $v_{N}^{i}(x)=\left(K-e^{x}\right)_{+}$. For $s=N-1, N-2, \ldots, 0$, set $\Delta_{s}=t_{s+1}-t_{s}, q^{s}=r+\left(\Delta_{s}\right)^{-1}$, and denote by $v_{s}^{i}(x)$ Carr's randomised approximation to $v^{i}\left(t_{s}, x\right)$.

The early exercise boundary $h_{s}^{i}$ for an interval $\left(t_{s}, t_{s+1}\right)$ and $v_{s}^{i}(x)$ can be found using backward induction. For $s=N-1, N-2, \ldots$, the boundary $h_{s}^{i}$ is chosen to maximise

$$
v_{s}^{i}(x)=E\left[\int_{0}^{\tau_{s}^{i}} e^{-q^{s} t} v_{s+1}^{i}\left(X_{t}^{0, x}\right) d t\right]+E\left[e^{-q^{s} \tau_{s}^{i}} \phi_{s}\left(X_{\tau_{s}^{i}}^{0, x}\right)\right],
$$

where $\tau_{s}^{i}$ is the hitting time of the interval of the form $\left(-\infty, h_{s}^{i}\right]$, and

$$
\phi_{s}^{(i)}(x)=\left(K-e^{x}\right)+E\left[e^{-r \delta} v^{(i-1)}\left(t_{s}+\delta, X_{t_{s}+\delta}^{t_{s}, x}\right)\right], \quad t_{s} \leq T-\delta,
$$

and

$$
\phi_{s}^{(i)}(x)=\left(K-e^{x}\right), \quad t_{s}>T-\delta .
$$

As in Boyarchenko and Levendorskii (2009), where the case of American options was considered, to derive (5.1), we replace $\phi(x)=\left(K-e^{x}\right)_{+}$in $(3.1)$ with $\left(K-e^{x}\right)$. This replacement is justified by a simple consideration that it is non-optimal to exercise the option when $\left(K-e^{x}\right) \leq 0$.

In the paper, we use uniform spacing; therefore, $q^{s}$ and $\Delta_{s}$ are independent of $s$ and denoted $q$ and $\Delta t$, respectively. For the case of put swing options, $v_{s}^{i}$ given by (5.1) is a unique solution of the boundary problem

$$
\begin{aligned}
(q-L) v_{s}^{i}(x) & =(\Delta t)^{-1} v_{s+1}^{i}(x), & & x>h_{s}^{i} \\
v_{s}^{i}(x) & =\phi_{s}^{(i)}(x), & & x \leq h_{s}^{i} .
\end{aligned}
$$

Note that the problem (5.2)-(5.3) can be obtained by discretisation of the time derivative in the generalised Black-Scholes equation (see details in Boyarchenko and Levendorskii (2009) and the bibliography therein).

Let the refracting period $\delta$ be equal to $k \Delta t$, where $k$ is a certain positive integer. Then, for $i=1, \ldots, n$,

$$
\phi_{s}^{(i)}(x)=\left(K-e^{x}\right)+u_{s}^{i-1}(x)
$$


where

$$
\begin{aligned}
& u_{s}^{0}(x)=0 \\
& u_{s}^{i}(x)=0, \quad t_{s}>T-\delta \\
& u_{s}^{i}(x)=E\left[e^{-r \delta} v_{s+k}^{(i)}\left(X_{t_{s+k}}^{t_{s} x}\right)\right], \quad t_{s} \leq T-\delta .
\end{aligned}
$$

Introduce $\tilde{v}_{s}^{i}(x)=v_{s}^{i}(x)-\phi_{s}^{(i)}(x)$, and substitute $v_{s}^{i}(x)=\tilde{v}_{s}^{i}(x)+\phi_{s}^{(i)}(x)$ into (5.2)-(5.3) as follows:

$$
\begin{aligned}
(q-L) \tilde{v}_{s}^{i}(x) & =(\Delta t)^{-1} G_{s}^{i}(x), \quad x>h_{s}^{i}, \\
\tilde{v}_{s}^{i}(x) & =0, \quad x \leq h_{s}^{i},
\end{aligned}
$$

where $G_{s}^{i}=\tilde{v}_{s+1}^{i}+\phi_{s+1}^{(i)}-\Delta t(q-L) \phi_{s}^{(i)}$.

Using similar arguments to those by Boyarchenko and Levendorskii (2009), it can be shown that for $s=n-1, n-2, \ldots, 0$, the function $G_{s}^{i}$ is a non-decreasing continuous function satisfying bound (2.18) with $\sigma_{+}=1, \sigma_{-}=0$; in addition,

$$
G_{s}^{i}(-\infty)<0<G_{s}^{i}(+\infty)=+\infty
$$

Then $G_{s}^{i}(x)$ satisfies the conditions of Theorem 2.6 (Boyarchenko and Levendorskii (2009)). Due to this theorem and to Proposition 2.1, we obtain that the following statements hold:

1. the function

$$
\tilde{w}_{s}^{i}:=\mathcal{E}_{q}^{+} G_{s}^{i}
$$

is continuous; it increases and satisfies (5.10);

2. the equation

$$
\tilde{w}_{s}^{i}(h)=0
$$

has a unique solution, denoted by $h_{s}^{i}$;

3. the hitting time of $\left(-\infty, h_{s}^{i}\right], \tau\left(h_{s}^{i}\right)$ is a unique optimal stopping time;

4. (Carr's approximation to) the swing option value with $i$ exercise rights at the moment $s$ is given by

$$
v_{s}^{i}=(q \Delta t)^{-1} \mathcal{E}_{q}^{-} \mathbf{1}_{\left(h_{s}^{i},+\infty\right)} \tilde{w}_{s}^{i}+\phi_{s}^{(i)}
$$

equivalently,

$$
\tilde{v}_{s}^{i}=(q \Delta t)^{-1} \mathcal{E}_{q}^{-} \mathbf{1}_{\left(h_{s}^{i},+\infty\right)} \tilde{w}_{s}^{i}
$$

5. $\tilde{v}_{s}^{i}=v_{s}^{i}-\phi_{s}^{(i)}$ is a positive non-decreasing function that admits bound (2.18) with $\sigma_{+}=1, \sigma_{-}=0$ and satisfies $\tilde{v}_{s}^{i}(+\infty)=+\infty$; it vanishes below $h_{s}^{i}$ and increases on $\left[h_{s}^{i},+\infty\right)$. 
Because functions $G_{s}^{i}$ and $\tilde{w}_{s}^{i}$ tend to plus infinity as $x \rightarrow+\infty$, the numerical calculation of the integrals in (5.11), (5.13) may face certain difficulties. To improve the convergence, we reformulate the algorithm in terms of the bounded functions $v_{s}^{i}$. Taking into account (5.4) and (2.5), $G_{s}^{i}$ can be rewritten as follows:

$$
\begin{aligned}
G_{s}^{i}(x) & =v_{s+1}^{i}(x)-\Delta t(q-L) u_{s}^{(i-1)}(x)-\Delta t(q-L)\left(K-e^{x}\right) \\
& =v_{s+1}^{i}(x)-\tilde{u}_{s}^{(i-1)}(x)-\left(\Delta t K q-e^{x}\right)
\end{aligned}
$$

where $\tilde{u}_{s}^{i}(x)$ can be approximated by the formulae

$$
\begin{aligned}
& \tilde{u}_{s}^{0}(x)=0 ; \\
& \tilde{u}_{s}^{i}(x)=0, \quad t_{s}>T-\delta \\
& \tilde{u}_{s}^{i}(x)=E\left[e^{-r(\delta-\Delta t)} v_{s+k}^{(i)}\left(X_{t_{s+k}}^{t_{s+1}, x}\right)\right]+o(\Delta t), \quad t_{s} \leq T-\delta .
\end{aligned}
$$

Notice that we can easily compute the expectation in the RHS of (5.18) using the Fourier transform technique (see, e.g., Carr and Madan (1999) or Boyarchenko and Levendorskii (2002)) as follows:

$$
\tilde{u}_{s}^{i}(x) \approx(2 \pi)^{-1} e^{-\rho x} \int_{-\infty}^{+\infty} e^{i x \xi-(\delta-\Delta t)(r+\psi(\xi+i \rho))} \hat{v}_{s+k}^{(i), \rho}(\xi) d \xi
$$

where $\hat{v}_{s}^{(i), \rho}(\xi)$ is the Fourier transform of the price $v_{s}^{(i)}(x)$ multiplied by an appropriate damping exponential factor $e^{\rho x}$; in our case, $\rho>0$. Numerically, the formula (5.19) can be efficiently realised by means of the FFT technique (cf. Carr and Madan (1999)).

However, for very short refracting periods $\delta$, the integrand in (5.19) may decay slowly at infinity (see e.g. Lord et al. (2008)). Hence, the numerical implementation of the Fourier transform may not be sufficiently accurate. To circumvent the potential numerical pricing difficulties when dealing with the case $\delta=k \Delta t, k>1$, ( $k$ is not too large and $\Delta t$ is small), the finite difference approach proposed in Section 4 can be used efficiently to find $\tilde{u}_{s}^{i}(x)$. The integral in (5.19) can be interpreted as the solution at time $\Delta t$ to the problem (4.2), with $t_{s}$ instead of $t$. Finally, if $\delta=\Delta t$, then $\tilde{u}_{s}^{i}(x) \approx v_{s+k}^{(i)}(x)$.

Now, we can rewrite (5.13) as follows:

$$
v_{s}^{i}=(q \Delta t)^{-1} \mathcal{E}_{q}^{-}\left(\mathbf{1}_{\left(h_{s}^{i},+\infty\right)} w_{s}^{i}-\mathbf{1}_{\left(-\infty ; h_{s}^{i}\right]} w_{s, 0}^{i}\right),
$$

where

$$
\begin{aligned}
w_{s}^{i} & =\mathcal{E}_{q}^{+} v_{s+1}^{i} ; \\
w_{s, 0}^{i} & =\mathcal{E}_{q}^{+}\left(\tilde{u}_{s}^{(i-1)}(x)+\Delta t K q-e^{x}\right)=\mathcal{E}_{q}^{+} \tilde{u}_{s}^{(i-1)}(x)+\Delta t K q-\varphi_{q}^{+}(-i) e^{x},
\end{aligned}
$$

and $h_{s}^{i}$ is a solution to the equation

$$
w_{s}^{i}=w_{s, 0}^{i} .
$$

Notice that in (5.20) and (5.21), the functions in the arguments of the operators $\mathcal{E}_{q}^{-}$and $\mathcal{E}_{q}^{+}$are bounded. The algorithm can be efficiently realised by using the Fast Wiener-Hopf factorisation method (see details in Section 2, Kudryavtsev and Levendorskii (2009)). 


\section{Numerical results}

In this section, we numerically illustrate the efficiency and the robustness of the proposed methods using the parameters of the numerical examples for pricing swing options in the Black-Scholes and CGMY models provided in Wilhelm and Winter (2008).

We consider a put swing option with $n=1,2,3$ exercise numbers and a refracting period $\delta=0.1$. We assume that the initial value of the stock prices is $S=100$, the exercise price $K=100$, the maturity $T=1$, and the force of the interest rate $r=0.05$.

In order to solve numerically the PIDE by using the finite difference scheme, we first localise the variables and the integral term to bounded domains. We use for this purpose the estimates for the localisation domain and truncation of large jumps given in Voltchkova and Tankov (2008).

In the case of Wiener-Hopf approach, we use adaptive method from Kudryavtsev and Levendorskii (2009). For a fixed number of time steps, $N$, and step in $x$-space, $\Delta x$, we increase the domain in $x$ space two-fold to ensure that the price does not change significantly. In the dual space it corresponds to increasing the number of points $M$. Fix the space step $\Delta x>0$ and the number of space points (and dual space points) $M=2^{m}$. Define the partitions of the normalised log-price domain $\left[-\frac{M \Delta x}{2} ; \frac{M \Delta x}{2}\right)$ by points $x_{k}=-\frac{M \Delta x}{2}+k \Delta x, k=0, \ldots, M-1$, and the frequency domain $\left[-\frac{\pi}{\Delta x} ; \frac{\pi}{\Delta x}\right]$ by points $\xi_{l}=\frac{2 \pi l}{h \Delta x}$, $l=-M / 2, \ldots, M / 2$.

In our examples both methods use a spatial discretisation step $\Delta x=0.001$ and a varying number of time steps $N=50,100,200$. In the Wiener-Hopf approach, the optimal choice of the number of the space points is $M=4096$ (the doubling the number $M$ changes the option prices by $0.0001 \%$ or less).

We propose first to assess the numerical robustness of our algorithm in the Black-Scholes case, using the volatility $\sigma=0.3$. In Table 1 , we report the prices (with time in seconds in parentheses) with the relative errors in a Black-Scholes framework, using the finite difference method (FD) proposed in Section 4 and the Wiener-Hopf approach (FWHF) proposed in Section 5. As benchmark solutions, we take the ones provided in Wilhelm and Winter (2008) (B-WW).

Table 1: Swing options prices in the Black-Scholes model

\begin{tabular}{c|c|c|c|c|c|c|c|} 
& & \multicolumn{5}{|c|}{ Prices } & \multicolumn{3}{|c|}{ Relative errors } \\
\cline { 3 - 8 } & $\mathrm{N}$ & $\mathrm{n}=1$ & $\mathrm{n}=2$ & $\mathrm{n}=3$ & $\mathrm{n}=1$ & $\mathrm{n}=2$ & $\mathrm{n}=3$ \\
\hline \multirow{3}{*}{ FWHF } & 50 & $9.786(0.22)$ & $19.130(0.7)$ & $27.968(1.17)$ & $-0,85 \%$ & $-0,65 \%$ & $-0,56 \%$ \\
& 100 & $9.826(0.42)$ & $19.190(1.38)$ & $28.045(2.33)$ & $-0,45 \%$ & $-0,34 \%$ & $-0,29 \%$ \\
& 200 & $9.848(0.83)$ & $19.222(2.72)$ & $28.085(4.59)$ & $-0,22 \%$ & $-0,17 \%$ & $-0,15 \%$ \\
\cline { 2 - 8 } & $\mathrm{B}-\mathrm{WW}$ & 9.8700 & 19.2550 & 28.1265 & - & - & - \\
\hline \multirow{2}{*}{ FD } & 50 & $9.834(0.12)$ & $19.096(5.16)$ & $27.711(8.10)$ & $-0,36 \%$ & $-0,83 \%$ & $-1,48 \%$ \\
& 100 & $9.864(0.27)$ & $19.184(8.64)$ & $27.925(16.47)$ & $-0,06 \%$ & $-0,37 \%$ & $-0,72 \%$ \\
& 200 & $9.867(0.57)$ & $19.220(16.68)$ & $28.027(33.0)$ & $-0,03 \%$ & $-0,18 \%$ & $-0,35 \%$ \\
\hline
\end{tabular}

Furthermore, we provide numerical results in a Lévy market model. To be exact, we use the 
CGMY model (Carr et al. 2002) with $C=1, G=10, M=10, Y=0.5$. No comparison results are available in the paper by Wilhelm and Winter (2008), and thus, we use as the benchmark value the FWHF method with a very fine mesh grid $(\Delta x=0.0002, N=800$ and $M=32768$ ), so that the doubling the number $N$, decreasing the space step twice and increasing the number of points 4 -fold change the option prices by $0.02 \%$ or less. In Table 2 , we report the numerical results for the CGMY model.

Table 2: Swing options prices in the CGMY model

\begin{tabular}{c|c|c|c|c|c|c|c|} 
& \multirow{4}{*}{} & \multicolumn{4}{|c|}{ Prices } & \multicolumn{3}{|c|}{ Relative errors } \\
\cline { 3 - 8 } & $\mathrm{N}$ & $\mathrm{n}=1$ & $\mathrm{n}=2$ & $\mathrm{n}=3$ & $\mathrm{n}=1$ & $\mathrm{n}=2$ & $\mathrm{n}=3$ \\
\hline \multirow{3}{*}{ FWHF } & 50 & $7.100(0.22)$ & $13.859(0.7)$ & $20.228(1.17)$ & $-0,80 \%$ & $-0,61 \%$ & $-0,54 \%$ \\
& 100 & $7.131(0.42)$ & $13.905(1.38)$ & $20.287(2.33)$ & $-0,36 \%$ & $-0,28 \%$ & $-0,25 \%$ \\
& 200 & $7.147(0.83)$ & $13.928(2.72)$ & $20.317(4.59)$ & $-0,14 \%$ & $-0,11 \%$ & $-0,10 \%$ \\
\cline { 2 - 8 } & $\mathrm{B}-\mathrm{FWHF}$ & 7.157 & 13.944 & 20.337 & - & - & - \\
\hline \multirow{2}{*}{ FD } & 50 & $7.173(1.20)$ & $13.887(37.1)$ & $20.102(76.1)$ & $0,22 \%$ & $-0,41 \%$ & $-1,16 \%$ \\
& 100 & $7.172(2.31)$ & $13.928(146)$ & $20.238(286.5)$ & $0,21 \%$ & $-0,11 \%$ & $-0,49 \%$ \\
& 200 & $7.171(4.56)$ & $13.948(751)$ & $20.306(1398)$ & $0,20 \%$ & $0,03 \%$ & $-0,15 \%$ \\
\hline
\end{tabular}

Table 3 reports the prices of swing options in the CGMY model with decreasing values of refracting periods $\delta=0.1,0.01,0.001,0$. The prices are calculated using the FWHF method with a spatial discretisation step $\Delta x=0.001$ and a number of time steps $N=1000$; the other parameters remain the same. We see that the sequence of the prices grows up to the limit value as the refracting period goes to 0 . The limit value is the solution to the problem (3.2) with the reward function in (3.3) defined for zero refracting period. Hence, in the limit case, we obtain a simplified problem, because we do not need to calculate the expectation in (3.3).

Table 3: Convergence of swing options prices in the CGMY model

\begin{tabular}{c|c|c|}
\cline { 2 - 3 } \multicolumn{1}{c|}{} & $\delta$ & $\mathrm{n}=3$ \\
\hline FWHF & 0.1 & 20.3416 \\
& 0.01 & 21.3701 \\
& 0.001 & 21.4699 \\
\cline { 2 - 3 } & 0. & 21.4760 \\
\hline
\end{tabular}

All computations were performed in double precision on an Eee PC with the following characteristics: CPU Atom N450, $1.67 \mathrm{Ghz}, 2 \mathrm{~Gb}$ of RAM.

The numerical results confirm the reliability of both approaches showing the robustness of the methods. In particular, the Wiener-Hopf approach is undoubtedly a very precise and efficient method for pricing swing options in the presence of multiple jumps. 


\section{Acknowledgements}

The first author gratefully acknowledges financial support from the European Science Foundation (ESF) through the Short Visit Grant number 3404 of the program "Advanced Mathematical Methods for Finance" (AMaMeF).

\section{References}

[1] Barles, G., Daher, C. and Romano, M. Convergence of numerical Schemes for problems arising in Finance theory. Mathematical models and methods in applied Sciences, 1995, 5(1), 125-143.

[2] Barndorff-Nielsen, O. E. Processes of Normal Inverse Gaussian Type. Finance and Stochastics, 1998, 2, 41-68.

[3] Barndorff-Nielsen, O. E. and Levendorskii, S. Feller Processes of Normal Inverse Gaussian type. Quantitative Finance, 2001, 1, 318-331.

[4] Barrera-Esteve, C., Bergeret, F., Dossal, C., Gobet, E., Meziou, A., Munos, R., Reboul-Salze, D. Numerical methods for the pricing of swing options: a stochastic control approach. Methodol. Comput. Appl. Probab., 2006, 8(4), 517-540.

[5] Bouchard, B., El Karoui, N. and Touzi, N. Maturity randomization for stochastic control problems. Ann. Appl. Prob., 2005, 15(4), 2575-2605.

[6] Boyarchenko, S. I., and Levendorskii, S. Z. Option pricing for truncated Lévy processes. International Journal of Theoretical and Applied Finance, 2000, 3, 549-552.

[7] Boyarchenko, S. I., and Levendorskii, S. Z. Non-Gaussian Merton-Black-Scholes theory, World Scientific, New Jercey, London, Singapore, Hong Kong. 2002.

[8] Boyarchenko, S.I. and Levendorskii, S.Z. American options: the EPV pricing model. Annals of Finance, 2005, 1(3), 267-292.

[9] S.I. Boyarchenko and S.Z. Levendorskii, General Option Exercise Rules, with Applications to Embedded Options and Monopolistic Expansion, Contributions to Theoretical Economics, 2006, 6(1), Article 2.

[10] Boyarchenko S.I. and Levendorskii, S.Z. Pricing American options in regime-switching models. SIAM J. Control Optim., 2009, 48, 1353-1376.

[11] Briani, M., La Chioma, C. and Natalini, R. Convergence of numerical schemes for viscosity solutions to integro-differential degenerate parabolic problems arising in financial theory. Numer. Math., 2004, 98(4), 607-646. 
[12] Carmona, C. and Touzi, N. Optimal Multiple Stopping and Valuation of Swing Options. Mathematical Finance, 2008, 18(2), 239-268.

[13] Carr, P. and Faguet, D. Fast accurate valuation of American options, 1994. Working paper, Cornell University, Ithaca.

[14] Carr, P. Randomization and the American put. Review of Financial Studies, 1998, 11, 597-626.

[15] Carr, P., Madan, D. Option valuation using the Fast Fourier Transform. Journal of Computational Finance.- 1999. - Vol. 3.- P. 463-520.

[16] Carr, P., Geman, H., Madan, D.B. and Yor, M. The fine structure of assets return: an empirical investigation. Journal of Business, 2002, 75(3), 305-332.

[17] Cont, R. and Voltchkova, E. A finite difference scheme for option pricing in jump-diffusion and exponential Lévy models. SIAM Journal on Numerical Analysis. 2005, 43(4), 1596-1626.

[18] Eberlein, E., and Keller, U. Hyperbolic distributions in finance. Bernoulli, 1995, 1, 281-299.

[19] Jaillet, P., Ronn, E.I. and Tompaidis, S. Valuation of Commodity-Based Swing Options. Management Science, 2004, 50, 909-921.

[20] Koponen, I. Analytic approach to the problem of convergence of truncated Lévy flights towards the Gaussian stochastic process. Physics Review E, 1995, 52, 1197-1199.

[21] Kou, S.G. A jump-diffusion model for option pricing. Management Science, 2002, 48, 1086-1101.

[22] Kudryavtsev, O.E. and Levendorskii, S.Z. Fast and accurate pricing of barrier options under Levy processes. Finance and Stochastics, 2009, 13(4), 531-562.

[23] R. Lord, F. Fang, F. Bervoets, and C.W. Oosterlee. A fast and accurate FFT-based method for pricing early-exercise options under L.evy processes. SIAM Journal on Scientific Computing, 30(4):1678-1705, 2008.

[24] Lari-Lavassani, A., Simchi, M. and Ware, A. A Discrete Valuation of Swing Options. Canadian Applied Mathematics Quarterly, 2001, 9(1), 35-73.

[25] Madan, D.B., Carr, P. and Chang, E.C. The variance Gamma process and option pricing. European Finance Review, 1998, 2, 79-105.

[26] Mnif, M. and Zhegal, A.B. Optimal Multiple stopping and Valuation of Swing Options in Lévy Models. International Journal of Theoretical and Applied Finance, 2006, 9(8), 1267-1298.

[27] PREMIA: An Option Pricer Project CERMICS - INRIA.

http://www.premia.fr 
[28] Press, W., Flannery, B., Teukolsky, S., Vetterling, W. Numerical recipes in C: The Art of Scientific Computing, Cambridge Univ. Press, Cambridge. 1992.

[29] Sato, K. Lévy processes and infinitely divisible distributions, Cambridge University Press, Cambridge. 1999.

[30] Voltchkova, E. and Tankov, P. Deterministic methods for option pricing in exponential Lévy models. Premia documentation: http://www.premia.fr, 2008.

[31] Wilhelm, M. and Winter, C. Finite element valuation of swing options. Journal of Computationl Finance, 2008, 11(3), 107-132. 